\title{
Using Bioinformatics To Mine The Hub Immune- Related Genes in Low-Grade Gliomas Based On TCGA and CGGA Databases
}

Chenyang Xu

Henan University

Song Li ( $\nabla$ hhlisong@henu.edu.cn )

Henan University https://orcid.org/0000-0001-7679-1548

Xiaodong Li

Henan University

Lei Gao

Henan University

\section{Primary research}

Keywords: Low grade gliomas, Immune-related score, Microenvironment, Prognostic, Bioinformatics

Posted Date: August 24th, 2021

DOI: https://doi.org/10.21203/rs.3.rs-637341/v1

License: (9) This work is licensed under a Creative Commons Attribution 4.0 International License. Read Full License 


\section{Abstract \\ Background}

Low grade gliomas(LGG) are the most common malignant tumors in the central nervous system. Tumor cells interact with immune cells and stromal molecules to form glioma immune microenvironment, which plays an important role in tumor progression.

\section{Methods}

According to the immune median score of ESTIMATE website, the gene expression and clinical data downloaded from TCGA database was analyzed in subgroups (high and low immune score groups). The DEGs were screened with the change threshold of $P<0.05$ and $|\log f \mathrm{c}| \geq 1$, and the volcano map and heat map were drawn by the ggplot2 package. Go and KEGG pathway enrichment analysis were performed by Metascape website. The PPI network of DEGs was constructed by STRING website. The hub genes were screened by using cytohubb plug-in of Cytoscape. CGGA and TCGA websites were utilized to analyze the overall survival of hub genes on LGG, and THPA database was used to further verify those protein expression in LGG and normal tissues.

\section{Results}

Subgroup analysis of immune-related score showed that the prognosis of patients was negatively correlated with immune-related score. The ROC curve of 5-year and 3-year survival rate was 0.88 and 0.75 respectively. The immune-related score was substantially correlated with IDH1 mutation status, tumor size and pathological type. The GO term enrichment showed that DEGs were closely related to the activation and regulation of immune related factors, the production and regulation of immune response. KEGG enriched in Staphylococcus aureus infection and phagosome. In addition, CPLX1 and TLR7 were associated with immune cell infiltration in tumor microenvironment.

\section{Conclusions}

Subgroups based on immune-related score can guide the prognosis of LGG. And CPLX1 and TLR7 may be the key genes of LGG immunology and potential biological targets for detection, diagnosis and immunology of LGG.

\section{Introduction}

The incidence rate and malignancy of glioblastoma multiforme (GBM) is the highest[1]. Low grade gliomas (LGG) often occur in supratentorial regions and account for about $5 \%$ of all primary brain tumors and $15 \%$ of all gliomas[2]. Low grade gliomas have malignant biological characteristics such as invasive 
growth, chemoradiotherapy resistance, and have a tendency to progress to high-grade gliomas. Traditional treatment methods include total tumor resection, postoperative radiotherapy, chemotherapy and other comprehensive treatment, but the prognosis of patients has not yet been ideal [3].

Glioma cells attract immune cells and stromal molecules into tumor tissue by secreting a variety of factors, and interact with tumor cells to form glioma immune microenvironment [4]. Tumor-associated macrophages (TAM), T cells and B cells are important components of glioma immune microenvironment [5]. Glioma cells secrete immunosuppressive factors such as interleukin-10 (IL-10) which form tumor immunosuppressive microenvironment and promote the invasion of tumor cells [6, 7]. Isocitrate dehydrogenase 1 and 2 (IDH1 and IDH2) are the key enzymes in the tricarboxylic acid cycle. The mutation of IDH 1/2 gene in tumor cells causes a large accumulation of 2-hydroxyglutaric acid [8]. Most of IDH mutations occurred in IDH 1 gene, and less than 1\% occurred in IDH 2 gene [9]. The mutation status of IDH $1 / 2$ gene in tumor cells is an essential reference index for LGG molecular typing. The prognosis of patients with IDH 1/2 mutant low grade glioma (IDH 1/2-mu LGG) is preferable to that of patients with IDH 1 / 2 wild type low grade glioma (IDH 1/2-wt LGG) [10]. IDH 1 mutation status of glioma cells affects the construction of glioma immune microenvironment [11], but its impact on the degree of immune invasion and functional status and the value of immune-score related indicators in predicting the prognosis of LGG molecular subtypes are not clear.

In this study, Using a variety of bioinformatics methods to mine and analyze LGG data in The Cancer Genome Atlas (TCGA), we revealed the expression differences of specific markers between subgroup of LGG immune-related score, screened out the key genes significantly related to tumor immune and patient prognosis, and explored their correlation with clinical parameters and IDH1 mutation status of patients. Finally, we utilized the Chinese glioma Genome Atlas Database (CGGA) and the Human Protein Atlas (THPA) to verify the protein expression and value of key genes in LGG.

\section{Materials And Methods}

\subsection{Expression profile and clinical data}

LGG gene expression data and clinical data such as case grade, mutation and survival time were collected from TCGA. Immune-related score data were obtained from the ESTIMATE website based on LGG gene expression data [12]. The data collected sequencing information of 425 LGG tissues, base on which we further constructed the immune risk score model and analyzed the correlation between the clinical parameters and the high/ low immune scores.

\subsection{DEGs analyses}

The gene expression and clinical data were divided into high and low score group based on the median immune-related score. The volcano map and heat map of the gene expression were made by $\mathrm{R}$ software, and the genes obtained after adjusted $P<0.05$ and $\log F C \geqq 1$ as significantly up-regulated $D E G s$, and adjusted $\mathrm{P}<0.05$ and $\log F C \leq-1$ as significantly down regulated $\mathrm{DEGs}$. Through the analysis tool 
Metascape (https://metascape.org/), Go and KEGG pathway of the DEGs were performed to collect the items with $\mathrm{P}<0.01$ and enrichment factor $>2.0$.

\subsection{PPI networks}

Proteins interact with each other to be involved in cell signal transduction, energy metabolism and material metabolism. In the protein-protein interaction network, some nodes interact with most proteins. These nodes are called hubs, which play more significant roles in the development of diseases. The PPI network of DEGs was constructed and visualized by STRING website (http://string-db.org) and Cytoscape software [13]. The six hub genes were screened by using cytoHubb plug-in of Cytoscape.

\subsection{Prognostic analyses and validation of key genes}

The CGGA contains the genomic data of nearly 2000 cases of glioma in different histopathological classification, malignancy grade and primary/recurrent of Chinese population. The overall survival (OS) of hub genes in LGG were performed based on TCGA and CGGA databases. Kaplan Meier method was used to draw survival time curve, and log rank test was used to verify. The correlation between gene expression and abundance of immune infiltrates via TIMER [14]. The stromal and immunes score of LGG were download by ESTIMATE. The human protein atlas (THPA) database was utilized to further verify its protein expression in LGG and normal tissues [15].

\subsection{Statistical analyses}

$R$ (version 3.5.2) software was used for statistical analysis and drawing. Wilcoxon rank sum test (FDR < 0.05, log2 | fold change | > 2) was used to screen the DEGs in LGG. In patients with overall survival (OS) > 3 months, the immune genes related to OS were obtained by Univariate analysis, and the immune genes that can independently affect the prognosis were screened by Multivariate analysis. T test and Pearson correlation test were used to analyze the relationship. $\mathrm{P}<0.05$ was considered as statistically significant.

\section{Results}

\subsection{Immune score and clinical prognosis analysis}

The gene expression profile and clinical information of LGG patients were matched with the immunerelated score (Table 1). According to the estimate algorithm, the clinical data were divided into high and low immune-related score groups. Kaplan Meier analysis was used to analyze high and low immune score (including stromal-score, immune-score and estimate-score) by log rank test. There was significant difference in survival prognosis between the two groups, especially in the aspect of ESTIMATE-score (Fig. 1). The ESTIMATE score is the sum of the immune score and the stromal score. The higher the immunity score, the worse the prognosis of patients. Combined with the results of multivariate analysis, immune-related score was an independent indicator of poor prognosis (Fig. 2A). The ROC curve for predicting 5-year and 3-year survival rate was 0.88 and 0.75 respectively, which indicated that the immune score had good predictive ability(Fig. 2B). Next, we compared the correlation analysis between 
immune score, stromal score and clinical parameters (IDH1 mutation status, tumor location, dimension and histological type). Immune-related score of IDH1 mutation groups was lower than those of IDH1 wild groups (Fig. 2C). Immune-related score of tumor located in temporal lobe group was higher than those of the other three groups(Fig. 2D). Interestingly, the immune-related score was positively correlated with tumor size (Fig. 2E). Immune-related score of patients with dimension larger than $1 \mathrm{~cm}$ were significantly higher than those of patients with dimension less than $0.5 \mathrm{~cm}$. Immune-related score of the pathological type of oligodendroglioma group was higher than those of astrocytoma groups(Fig. 2F). 
Table 1

Demographic and clinicopathological

characteristics of patients with LGG in TCGA database

\begin{tabular}{|c|c|}
\hline $\begin{array}{l}\text { Demographic or } \\
\text { Characteristics }\end{array}$ & $\begin{array}{l}\text { All subjects } \\
(\mathrm{N}=425)(\%)\end{array}$ \\
\hline \multicolumn{2}{|l|}{ Age } \\
\hline$\rrbracket=49$ & $291(68.4)$ \\
\hline $50-59$ & $84(19.7)$ \\
\hline $60-69$ & $40(9.4)$ \\
\hline$>=70$ & $10(2.3)$ \\
\hline \multicolumn{2}{|l|}{ Gender } \\
\hline Female & $225(52.9)$ \\
\hline Male & $200(47.1)$ \\
\hline \multicolumn{2}{|l|}{ Stromal_score } \\
\hline High & 212(49.9) \\
\hline Low & $213(50.1)$ \\
\hline \multicolumn{2}{|l|}{ Immune_score } \\
\hline High & $200(47.1)$ \\
\hline Low & $225(52.9)$ \\
\hline \multicolumn{2}{|l|}{ ESTIMATE_score } \\
\hline High & 212(49.9) \\
\hline Low & $213(50.1)$ \\
\hline \multicolumn{2}{|l|}{ Dimension(cm) } \\
\hline $0-0.49$ & $251(58.6)$ \\
\hline $0.5-1$ & $70(16.4)$ \\
\hline$>1$ & $104(24.4)$ \\
\hline \multicolumn{2}{|l|}{ Tumor_location } \\
\hline Supratentorial, Frontal Lobe & $247(58.1)$ \\
\hline Supratentorial, Occipital Lobe & 16(3.7) \\
\hline Supratentorial, Parietal Lobe & $489(80.9)$ \\
\hline
\end{tabular}




\begin{tabular}{|c|c|}
\hline \\
\hline $\begin{array}{l}\text { Demographic or } \\
\text { Characteristics }\end{array}$ & $\begin{array}{l}\text { All subjects } \\
(\mathrm{N}=425)(\%)\end{array}$ \\
\hline Supratentorial, Temporal Lobe & $426(70.5)$ \\
\hline \multicolumn{2}{|l|}{ IDH1_mutation } \\
\hline YES & 126(29.6) \\
\hline NO & $299(70.3)$ \\
\hline \multicolumn{2}{|l|}{ Histological_type } \\
\hline Astrocytoma & 157(36.9) \\
\hline Oligoastrocytoma & 106(24.9) \\
\hline Oligodendroglioma & 162(38.1) \\
\hline \multicolumn{2}{|l|}{ Karnofsky_performance_score } \\
\hline$\rrbracket=49$ & $157(36.9)$ \\
\hline $50-69$ & $34(8.0)$ \\
\hline$>=70$ & $234(55.0)$ \\
\hline
\end{tabular}

\subsection{DEGs analyses}

By comparing the gene expression profile data of 425 LGG patients between high and low ESTIMATEscore group, the gene expression threshold was adjusted and volcano map was drawn (Fig. 3A, B). The adjusted $P<0.05$ and $\square \log F C \unrhd \geqq 1$ were defined as significantly DEGs, and extracted 493 immune related DEGs. David was utilized to analyze the biological process of GO and KEGG pathway of the DEGs. Go term enrichment analysis showed that DEGs were closely related to the activation and regulation of cytokines, lymphocytes, T cells, leukocytes and other immune-related factors, as well as the production and regulation of immune response and cell adhesion, in which the number one is immune response and inflammatory response (Fig. 3C, 3D, 3E). KEGG analysis showed that DEGs were substantially enriched in Staphylococcus aureus infection and phagosome (Fig. 3F).

\subsection{Screening and prognostic analysis of hub genes}

The PPI network of DEGs was constructed by using the interactive gene retrieval tool string (Fig. 3S1), and then the most significant models were built by using the software Cytoscape (Fig. 3S2). Six hub genes were screened by the cytohubb plug-in of Cytoscape. The overall survival of the six hub genes based on gene expression in LGG patients was a significant statistical difference(Fig. 4A). The OS of elevated expression groups of CPLX1 and VSTM2A were higher than that of low expression groups, while the OS of high expression groups of CD300LF, IGSF6, ITGAM and TLR7 were significantly lower than that of low groups. Next, we used CGGA to verify the selected key genes, and the results showed that only CPLX1 and TLR7 were significantly correlated with overall survival of LGG patients(Fig. 4B). Next, we 
analyzed the relationship between CPLX1 and TLR7 and the clinicopathological characteristics of LGG patients(Fig. 5A), and showed that the expression of TLR7 gene in LGG tissue was significantly higher than that in normal tissue, while the expression of CPLX1 was contrary to the above results. Interestingly, TLR7 expression was positively correlated with LGG grade, and its expression was significantly higher in recurrent group than in primary tissue (Fig. 5B). On the contrary, CPLX1 was low expression of LGG tissues, which were negatively correlated with LGG grade, and its expression was low in the recurrent group.

In addition, we assessed the relationship between CPLX1 and TLR7 and the infiltration of immune cells (Fig. 5C). The results showed that the infiltration degree of B cells, CD4 + T cells, Macrophages and Dendritic cells were positively correlated with TLR7, while CPLX1 was negatively correlated with them. In order to verify the protein expression and localization of CPLX1 and TLR7 in LGG, the retrieval results of human protein expression map database showed that the expression abundance of CPLX1 protein in cancer tissues was significantly lower than that in normal tissues, while the expression abundance of TLR7 protein was highly expressed in cancer tissues (Fig. 6 ).

\section{Discussion}

Although some progress has been made in chemotherapy, radiotherapy and targeted therapy, the toxicity and side effects of therapeutic drugs, poor drug tolerance, high cost and many other factors have restricted the prognosis of LGG, resulting in high mortality [16]. In recent years, some studies and clinical trials have shown that immunotherapy has made progress in prolonging the remission rate and reducing the recurrence of LGG [17]. Immune process plays an important role in tumor invasion and drug resistance, and ultimately affects the therapeutic effect and clinical efficacy [18]. With the rapid progress and maturity of high-throughput sequencing, biochip and bioinformatics analysis, big data analysis, mining and development of key genes as biomarkers are used to predict different types of immunotherapy. This is a promising research field for LGG immunotherapy, and also provides new ideas for LGG pathogenesis and treatment evaluation.

Accurate prognosis prediction directly affects the choice of treatment for cancer patients. At present, the prognosis and treatment options of LGG patients are mainly based on stages and grades. However, there are still large differences in the prognosis of patients with similar stages and treatment methods [19]. Compared with IDH1 wild-type, patients with IDH1 gene mutation get a better prognosis [20]. Interestingly, according to the ESTIMATE algorithm and TCGA clinical prognosis data, we found that immune-related score was significantly correlated with clinical parameters (tumor location, dimension and histological type). Moreover, immune score and strong scores in IDH1 gene wild group were significantly higher than those in IDH1 mutation type. The ROC curve of the immune-related score for predicting the 5-year and 3year survival rate of patients was 0.88 and 0.75 respectively, indicating that the immune score has good predictive ability, suggesting that the immune score can assist clinical judgment of the prognosis of patients with glioma. 
In this study, we analyzed the gene expression profile of LGG patients in TCGA database by using estimate algorithm, and extracted 493 immune related DEGs related to survival. The important biological process of GO: DEGs is closely related to the activation and regulation of cytokines, lymphocytes, T cells, leukocytes and other immune related factors, as well as the production and regulation of immune response. KEGG enrichment of DEGs were remarkably in Staphylococcus aureus infection and phagosome. Therefore, we speculate that these DEGs play a key role in the microenvironment of immunosuppressed or activated tumor immune cells, and intervention of these immune targets may become an important target for tumor immunotherapy. In order to further explore the key genes in tumor microenvironment, we systematically explored the differentially expressed genes related to LGG immunity, and screened out 6 hub genes, and further analyzed and screened out 2 hub genes. Among them, TRL7and CPLX1 play a dominant role in the regulatory network in the progression of LGG.

TRL7 is not only expressed in immune cells, but also in tumor cells. Ochi et al. [21] found that TRL7 expression increased in human and mouse pancreatic cancer tissues, and stimulated by their ligands, the expression of P53, TGF- $\beta$ and c-Myc genes, which significantly accelerated tumor progression. TRL7 on lung cancer cell lines stimulated by corresponding ligands can activate NF-KB and up regulate the expression of $\mathrm{BCl}-2$ [22]. TRL7 was also found to be highly expressed in lung cancer [23]. These studies suggest that TRL7 may be closely related to the progression and development of the tumor. Our study showed that the OS of high expression group of TLR7 is lower than that of low expression group, which has been well verified in CGGA. The expression of TLR7 gene in LGG tissue is significantly higher than that in normal tissue. The expression of TLR7 is positively correlated with LGG grade, and its expression in the recurrent group is significantly higher than that in primary tissue. Interestingly, TLR7 is positively correlated with immune cell infiltration in tumor microenvironment. R848 is a synthetic guanine nucleotide analogue, and is the ligand of TLR7. It is currently used as a Th1 adjuvant in the treatment of viral infectious diseases and tumor [24]. R848 can increase its cytotoxicity and promote the production of related cytokines. Thus enhancing the body's immune response against pathogens and tumors [25]. This further confirmed that TLR7 as a target to regulate NK cell-mediated immune response may become a novel immunotherapy approach and method with a new application prospect.

Human Complexin (CPLX) is a presynaptic protein that widely exists in the presynaptic membrane and can affect synaptic transmission, including CPLX1 and CPLX2 [26, 27]. CPLX1 mainly exists in the granuloma cells and vertebral cells of the central nervous system, and is considered to be an axial tree type protrusion marker $[28,29]$. The expression of CPLX1 in LGG was markedly decreased, and it was related to patient grade and IDH1 mutation status. Interestingly, CPLX1 was negatively correlated with immune cell infiltration in tumor microenvironment. In this study, we found that the low expression of CPLX1 is closely related to the decrease of OS in LGG patients, which may become an effective indicator of the prognosis of LGG immunotherapy, which has not been reported in previous studies. Therefore, the molecular mechanism of CPLX1 affecting the adverse prognosis of LGG is worthy of further study.

To sum up, subgroups based on immune-related score can guide the prognosis of LGG. DEGs were mainly enriched in the immune regulation function of LGG patients, involving a variety of potential 
immune signal pathways. In addition, six LGG immune-related prognostic genes were obtained by survival analysis. In this study, we first analyzed the immunomodulation and prognosis of TRL7 and CPLX1 genes, and discussed their potential as biomarkers of LGG immune prognosis. These results suggest that TRL7 and CPLX1 may be the key genes of LGG immunotherapy, and may be potential biological targets for detection and immunotherapy of LGG.

\section{Abbreviations}

LGG

Low grade gliomas; GBM:glioblastoma multiforme; TAM:Tumor-associated macrophages;IDH1 and IDH2:Isocitrate dehydrogenase 1 and 2; TCGA:The Cancer Genome Atlas; ROC:receiver operating characteristic; DEGs:differentially expressed genes; TIMER:Tumor IMmune Estimation Resource; OS:overall survival; CGGA:the Chinese glioma Genome Atlas Database ; THPA:Human Protein Atlas.

\section{Declarations}

\section{Acknowledgements}

All authors declare that there are no conficts of interest.

\section{Authors' contributions}

Song Li put forward these ideas and designed the overall framework of this article. Xu assessed the data and wrote the article. Lei Gao downloaded and processed the original data and pictures. Xiaodong Li proofread, reviewed and considered the article.

\section{Founding}

This research was supported by the Project of Improving the Ability of Diagnosis and Treatment of Difficult Diseases(No.ZLYNXM202009) and the Science and Technology Research Project of Henan Province (No.182102311180).

\section{Availability of data and materials}

Not applicable.

\section{Ethics approval and consent to participate}

Not applicable.

\section{Consent for publication}

Not applicable. 


\section{Competing interests}

None declared.

\section{Author details}

${ }^{1}$ Neurosurgery department, Huaihe Hospital of Henan University, 8 Baobei Road, Gulou District, Kaifeng. China. ${ }^{2}$ Department of surgery, Huaihe hospital, Henan University, 8 Baobei Road, Gulou District, Kaifeng, Henan Province, China.

\section{References}

1. Dolecek TA, Propp JM, Stroup NE, Kruchko C. CBTRUS statistical report: Primary brain and central nervous system tumors diagnosed in the United States in 2005-2009. Neuro -Oncology. 2012; 14 Suppl 5: v1 -4 9.

2. Tandon A, Schiff D. Therapeutic decision making in patients with newly diagnosed low grade glioma. Curr Treat Options Oncol. 2014;15(4):529-38.

3. Nabors LB, Portnow J, Ammirati M, Baehring J, Engh AM. Central nervous system cancers, version 1. 2015 featured updates to the NCCN guidelines. J Natl Compr Cancer Netw. 2015;13(10):1191-202.

4. Orihuela R, Mcpherson CA, Harry GJ. Microglial M1/M2 polarization and metabolic states. Br J Pharmacol. 2016;173(4):649-65.

5. Hambardzumyan D, Gutmann $\mathrm{DH}$, Kettenmann $\mathrm{H}$. The role of microglia and macrophages in glioma maintenance and progression. Nat Neurosci. 2016;19(1):20-7.

6. Qi L, Yu H, Zhang Y, Zhao D, Lv P, Zhong Y, Xu Y. IL-10 secreted by M2 macrophage promoted tumorigenesis through interaction with JAK2 in glioma. Oncotarget. 2016;7(44):71673-85.

7. Ye XZ, Xu SL, Xin YH, Yu SC, Ping YF, Chen L, Xiao HL, Wang B, Yi L, Wang QL, Jiang XF, Yang L, Zhang P, Qian C, Cui YH, Zhang X, Bian XW. Tumor-associated microglia/macrophages enhance the invasion of glioma stem-like cells via TGF-beta1 signaling pathway. J Immunol. 2012;189(1):44453.

8. Dang L, White DW, Gross S, Bennett BD, Bittinger MA, Driggers EM, Fantin VR, Jang HG, Jin S, Keenan MC, Marks KM, Prins RM, Ward PS, Yen KE, Liau LM, Rabinowitz JD, Cantley LC, Thompson CB, Vander Heiden MG, Su SM. Cancer-associated IDH1 mutations produce 2-hydroxyglutarate. Nature. 2009;462(7274):739-44.

9. Hartmann C, Meyer J, Balss J, Capper D, Mueller W, Christians A, Felsberg J, Wolter M, Mawrin C, Wick W, Weller M, Herold-Mende C, Unterberg A, Jeuken JW, Wesseling P, Reifenberger G, von Deimling A. Type and frequency of IDH1 and IDH2 mutations are related to astrocytic and oligodendroglial differentiation and age: a study of 1,010 diffuse gliomas. Acta Neuropathol. 2009;118(4):469-74. 
10. Yan H, Parsons DW, Jin G, McLendon R, Rasheed BA, Yuan W, Kos I, Batinic-Haberle I, Jones S, Riggins GJ, Friedman H, Friedman A, Reardon D, Herndon J, Kinzler KW, Velculescu VE, Vogelstein B, Bigner DD. IDH1 and IDH2 mutations in gliomas. N Engl J Med. 2009;360(8):765-73.

11. Amankulor NM, Kim Y, Arora S, Kargl J, Szulzewsky F, Hanke M, Margineantu DH, Rao A, Bolouri H, Delrow J, Hockenbery D, Houghton AM, Holland EC. Mutant IDH1 regulates the tumor-associated immune system in gliomas. Genes Dev. 2017;31(8):774-86.

12. Yoshihara K, Shahmoradgoli M, Martínez E, Vegesna R, Kim H, Torres-Garcia W, Treviño V, Shen H, Laird PW, Levine DA, Carter SL, Getz G, Stemke-Hale K, Mills GB, Verhaak R. Inferring tumour purity and stromal and immune cell admixture from expression data. Nature Communications. $2013 ; 4$.

13. Shannon P, Markiel A, Ozier O, Baliga NS, Wang JT, Ramage D, Amin N, Schwikowski B, Ideker T. Cytoscape: a software environment for integrated models of biomolecular interaction networks. Genome Res. 2003;13(11):2498-504.

14. Li B, Severson E, Pignon JC, Zhao H, Li T, Novak J, Jiang P, Shen H, Aster JC, Rodig S, Signoretti S, Liu JS, Liu XS. Comprehensive analyses of tumor immunity: implications for cancer immunotherapy. Genome Biol. 2016;17(1):174.

15. Colwill K, Renewable Protein Binder Working Group, Gräslund S. A roadmap to generate renewable protein binders to the human proteome. Nat Methods. 2011;8(7):551-8.

16. Tandon A, Schiff D. Therapeutic decision making in patients with newly diagnosed low grade glioma. Curr Treat Options Oncol. 2014;15(4):529-38.

17. Järås $M$, Johnels $P$, Hansen $N$, Agerstam $H$, Tsapogas P, Rissler M, Lassen C, Olofsson T, Bjerrum OW, Richter J, Fioretos T. Isolation and killing of candidate chronic myeloid leukemia stem cells by antibody targeting of IL-1 receptor accessory protein. ProcNatl Acad Sci USA. 2010;107(37):162805 .

18. Smith DE, Hanna R, Della Friend, Moore H, Chen H, Farese AM, MacVittie TJ, Virca GD, Sims JE. The soluble form of IL-1 receptor accessory protein enhances the ability of soluble type II IL-1 receptor to inhibit IL-1 action. Immunity. 2003;18(1):87-96.

19. Scott R, Michal Z, Kohei F, Ana GS, Julie B, Anthony A, Paul K, Monique J, Alvaro L, Ute B. LGG-60. the genetic landscape of pediatric low-grade gliomas: incidence, prognosis and response to therapy. Neuro-Oncology.20(suppl_2):i117-i117.

20. Wang J, Yan L, Ai P, He Y, Guan H, Wei Z, He L, Mu X, Liu Y, Peng X. Observation versus radiotherapy with or without temozolomide in postoperative WHO grade II high-risk low-grade glioma: a retrospective cohort study. Neurosurg Rev. 2021;44(3):1447-55.

21. Ochi A, Graffeo CS, Zambirinis CP, Rehman A, Hackman M, Fallon N, Barilla RM, Henning JR, Jamal M, Rao R, Greco S, Deutsch M, Medina-Zea MV, Bin Saeed U, Ego-Osuala MO, Hajdu C, Miller G. Tolllike receptor 7 regulates pancreatic carcinogenesis in mice and humans. J Clin Invest. 2012;122(11):4118-29.

22. Cherfils-Vicini J, Platonova S, Gillard M, Laurans L, Validire P, Caliandro R, Magdeleinat P, MamiChouaib F, Dieu-Nosjean MC, Fridman WH, Damotte D, Sautès-Fridman C, Cremer I. Triggering of 
TLR7 and TLR8 expressed by human lung cancer cells induces cell survival and chemoresistance. J Clin Invest. 2010;120(4):1285-97.

23. Zhang YB, He FL, Fang M, Hua TF, Hu BD, Zhang ZH, Cao Q, Liu RY. Increased expression of Toll-like receptors 4 and 9 in human lung cancer. Molboil Rep. 2009;36(6):1475-81.

24. Toka FN, Nfon CK, Dawson H, Golde WT. Accessory- cell- mediated activation of porcine NK cells by toll- like receptor 7(TLR7) and TLR8 agonists. Clin Vaccine Immunol. 2009;16(6):866-78.

25. Bourquin C, Schmidt L, Lanz AL, Storch B, Wurzenberger C, Anz D, Sandholzer N, Mocikat R, Berger M, Poeck H, Hartmann G, Hornung V, Endres S. Immunostimulatory RNA oligonucleotides induce an effective antitumoral NK cell response through the TLR7. J Immunol. 2009;183(10):6078-86.

26. Tsuru E, Oryu K, Sawada K, Nishihara M, Tsuda M. Complexin 2 regulates secretion of immunoglobulin in antibody-secreting cells. Immunity, Inflammation and Disease. 2019; 7(4).

27. Courtney NA, Bao H, Briguglio JS, Chapman ER. Synaptotagmin 1 clamps synaptic vesicle fusion in mammalian neurons independent of complexin. Nature Communications. 2019; 10(1).

28. Eastwood SL, Harrison PJ. Synaptic pathology in the anterior cingulate cortex in schizophrenia and mood disorders. A review and a western blot study of synaptophysin, GAP-43 and the complexins. Brain Res Bull. 2001;55:569-78.

29. Tannenberg RK, Scott HL, Tannenberg AE, Dodd PR. Selective loss of synaptic proteins in Alzheimer's disease: evidence for an increased severity with APOE varepsilon4. Neurochem Int. 2006;49:631-9.

\section{Figures}


A

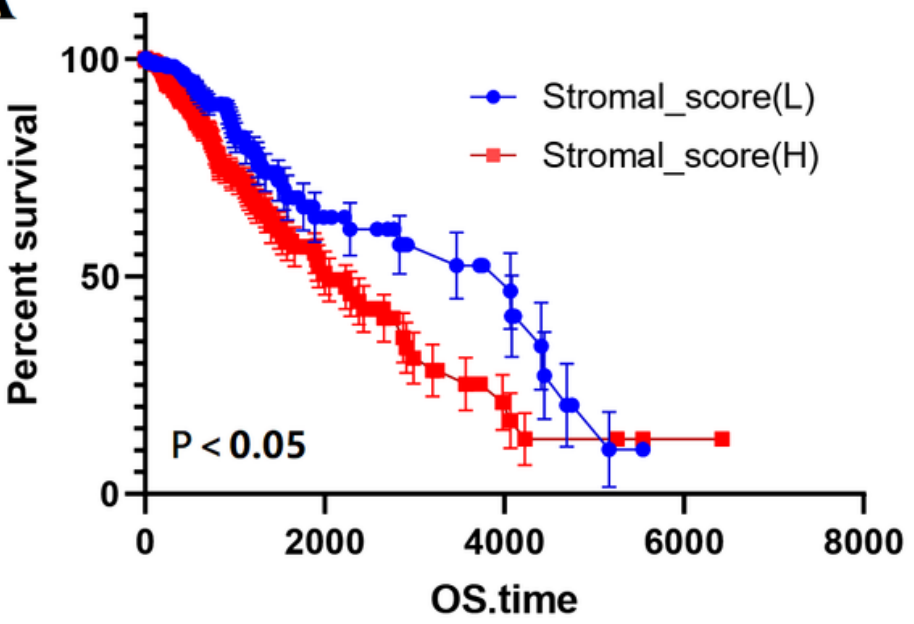

C

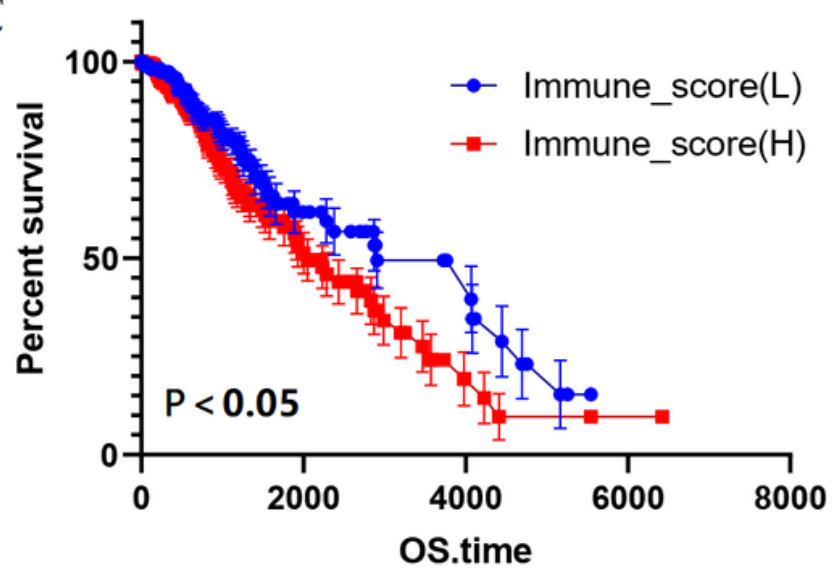

$\mathbf{E}$

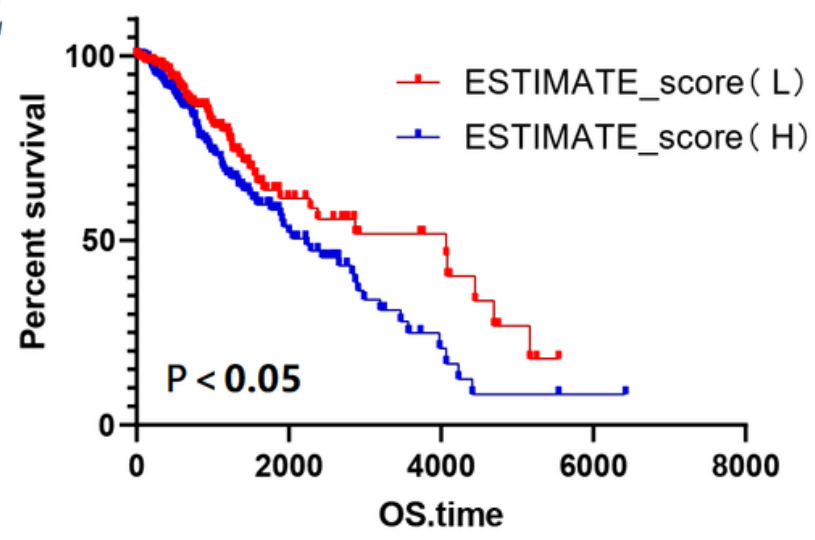

B

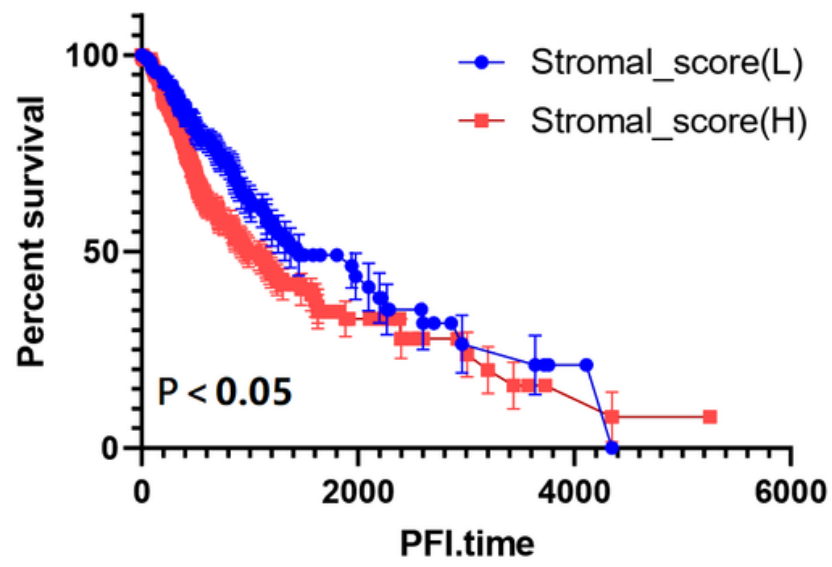

D

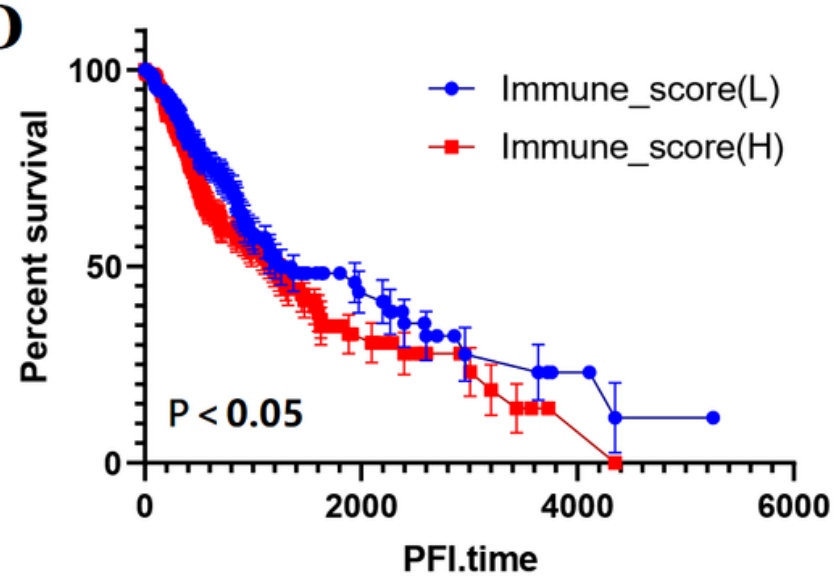

F

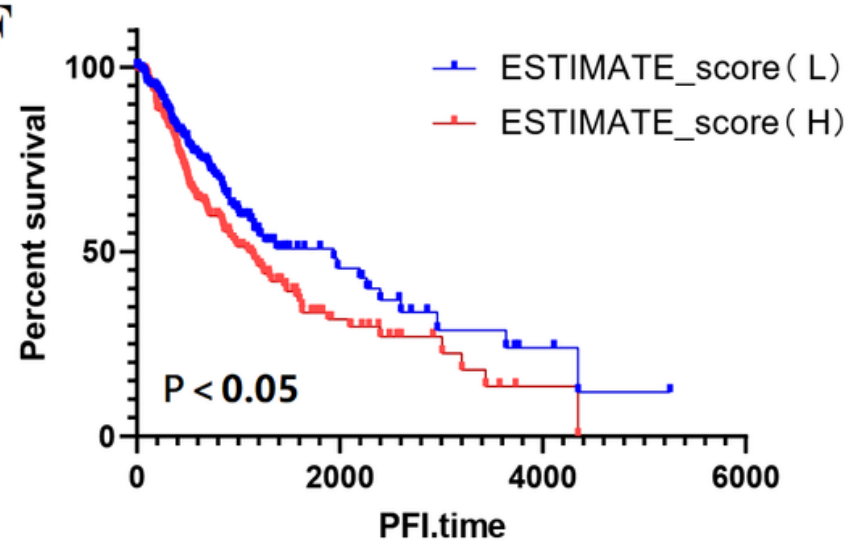

Figure 1

The OS (overall survival) and PFI (progression free interval) of LGG based on immune-related scores. The OS and RFS curves between high and low groups based on stromal scores(A, B). The OS and RFS curves between high and low groups based on immune scores(C, D). The OS and RFS curves between high and low groups based on ESTIMATE scores(E, F). 
A
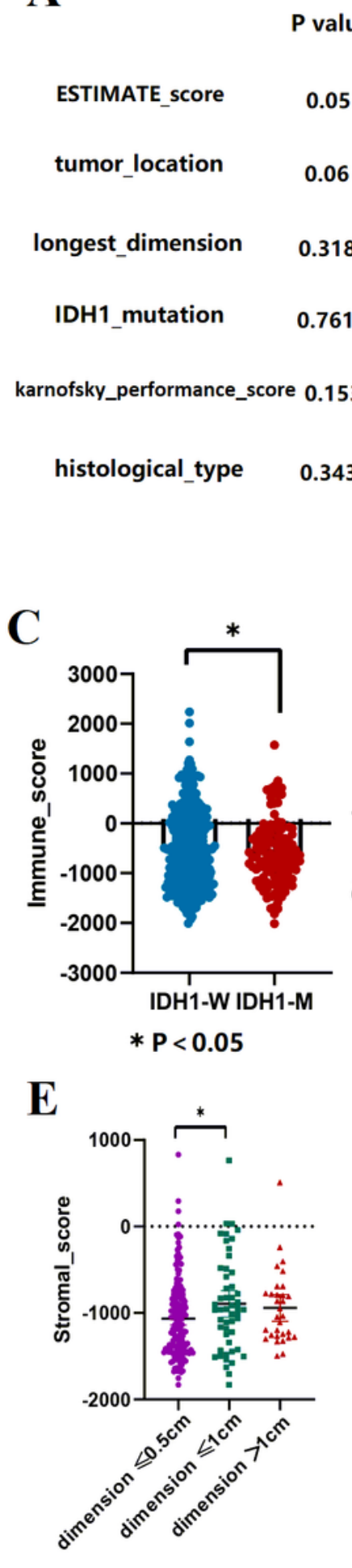

C
P value Hazard radio

$0.05 \quad 1.47(0.99-2.2)$

$0.06 \quad 1.12(0.99-1.21)$

$0.318 \quad 0.80(0.52-1.24)$

$0.761 \quad 0.92(0.55-1.54)$

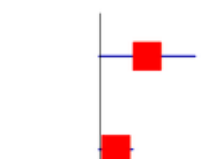

B

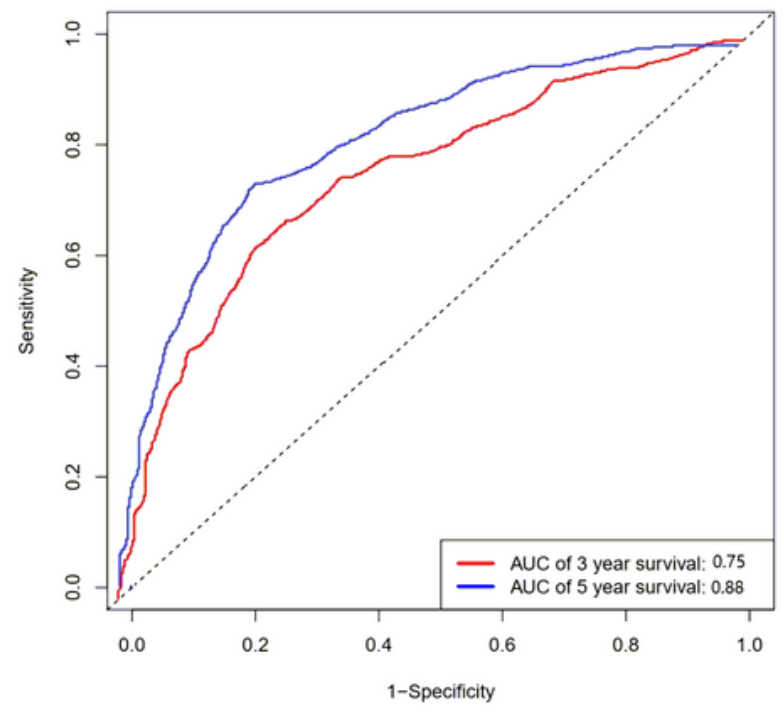

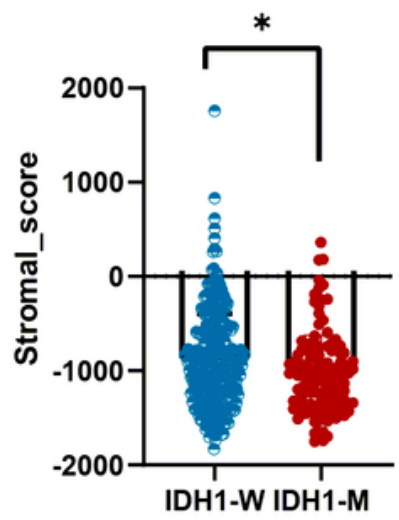

D

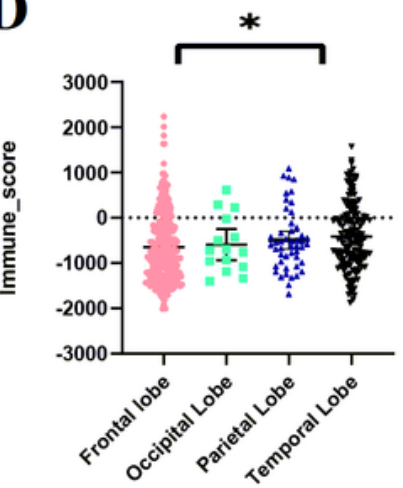

$\mathbf{F}$

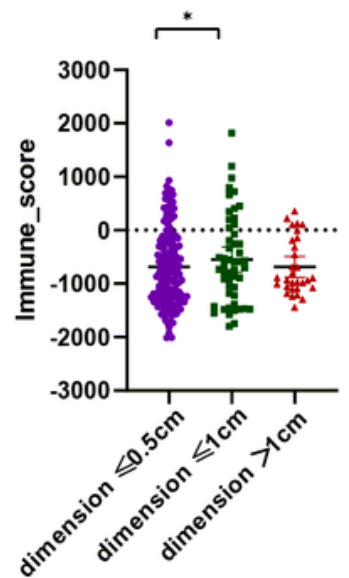

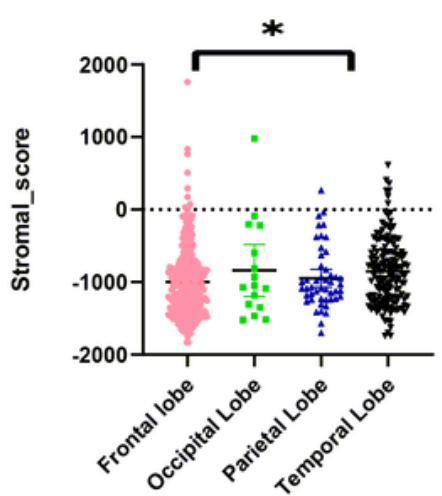

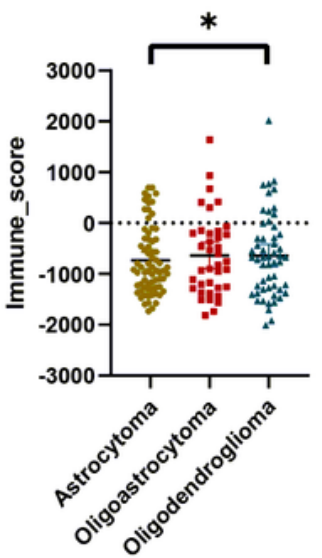

Figure 2

Correlation analysis between immune-related score and clinicopathological features. Multivariate analysis of factors including ESTIMATE scores, dimension, tumor location, IDH1_mutation, histological types and Karnofsky scores(A). The ROC curve for predicting 5-year and 3-year survival rate was 0.88 and 0.75 respectively $(\mathrm{B})$. The distribution of immune-related score in different clinicopathological features, including IDH1_mutation (C), tumor location(D), dimension(E) and histological type (F). 

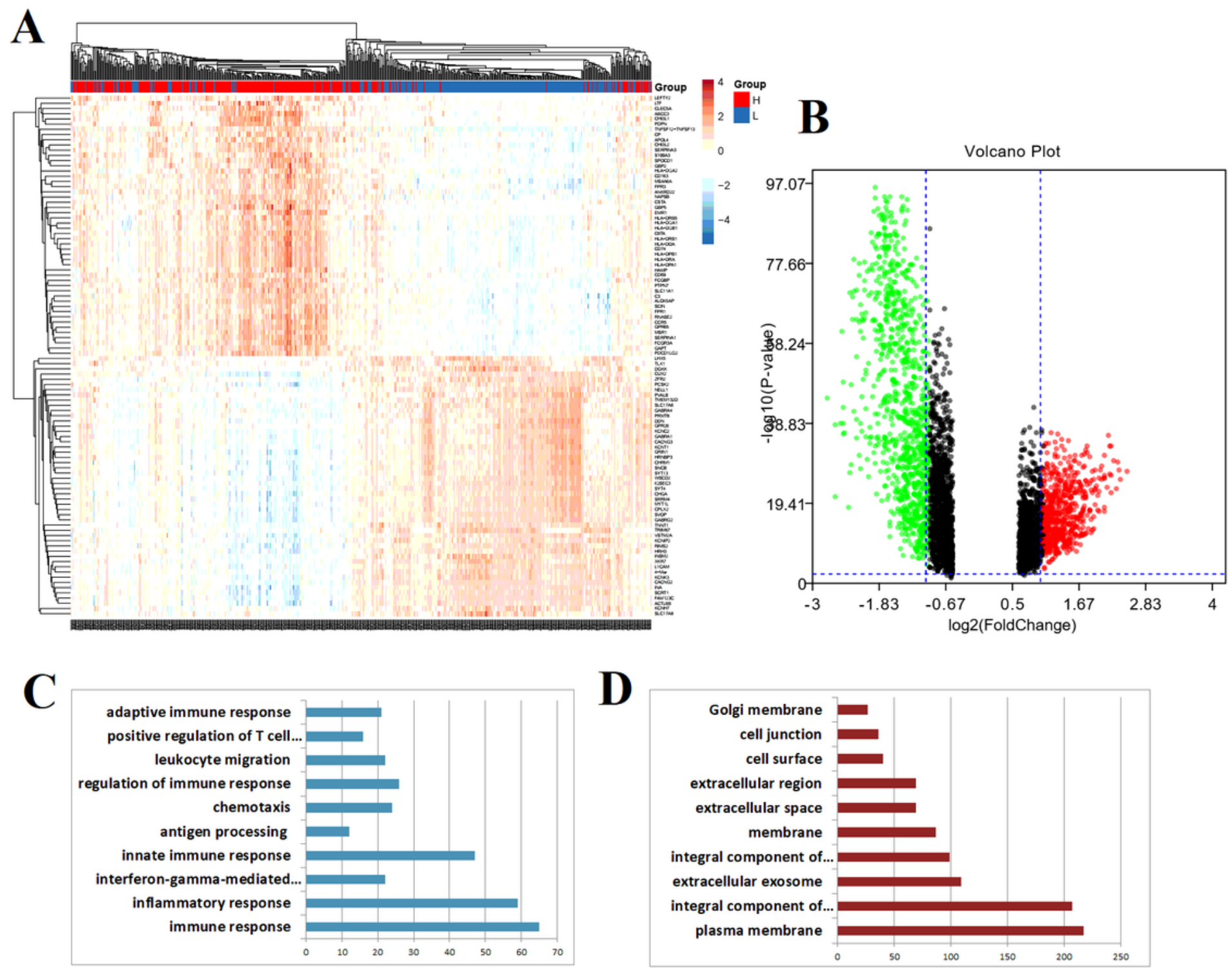

\section{$\mathbf{E}$}
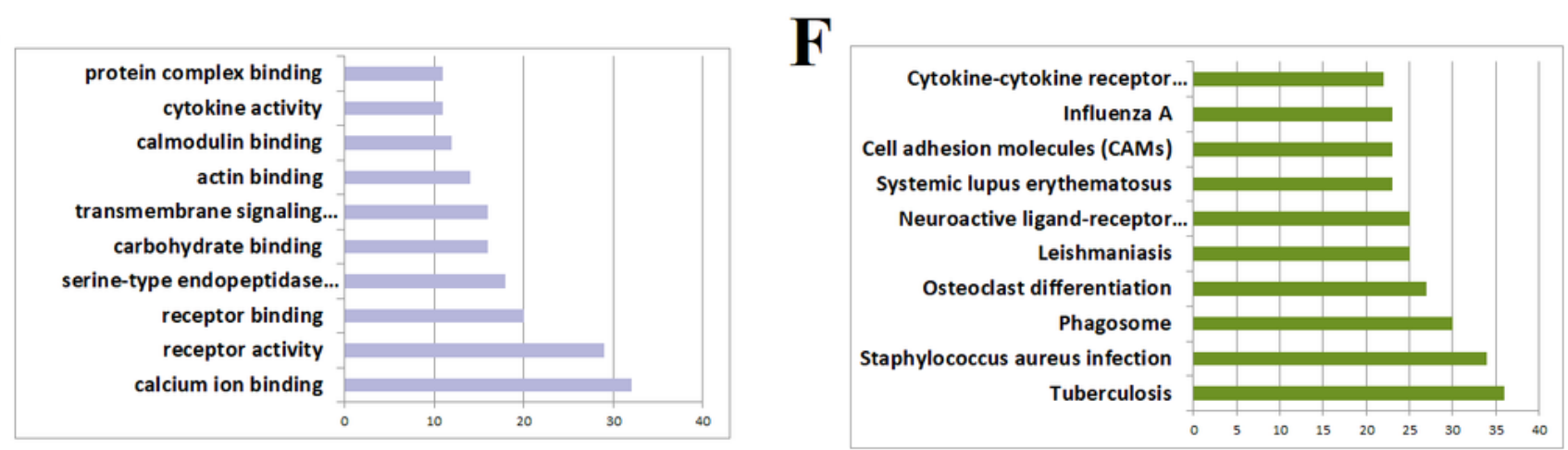

\section{Figure 3}

Screening and pathway analysis of DRGs. The heatmap was generated by the ggplot2 software package of R software. Heatmaps showed the different expression of genes between high and low groups of ESTIMATE scores (A). The gene expression threshold was adjusted and volcano map was drawn. The adjusted $P<0.05$ and $\log F C \geqq 1$ were defined as significantly up-regulated $D E G s$, and the adjusted $P<0.05$ and $\log F C \leq-1$ were defined as significantly down regulated DEGs (B). Go analysis is divided into three 
parts: Cellular Component (C), Molecular Function (D) and Biological Process (E). KEGG pathway enrichment analysis were performed to collect the items with $\mathrm{P}<0.01$ and enrichment factor $>2.0(\mathrm{~F})$.

A
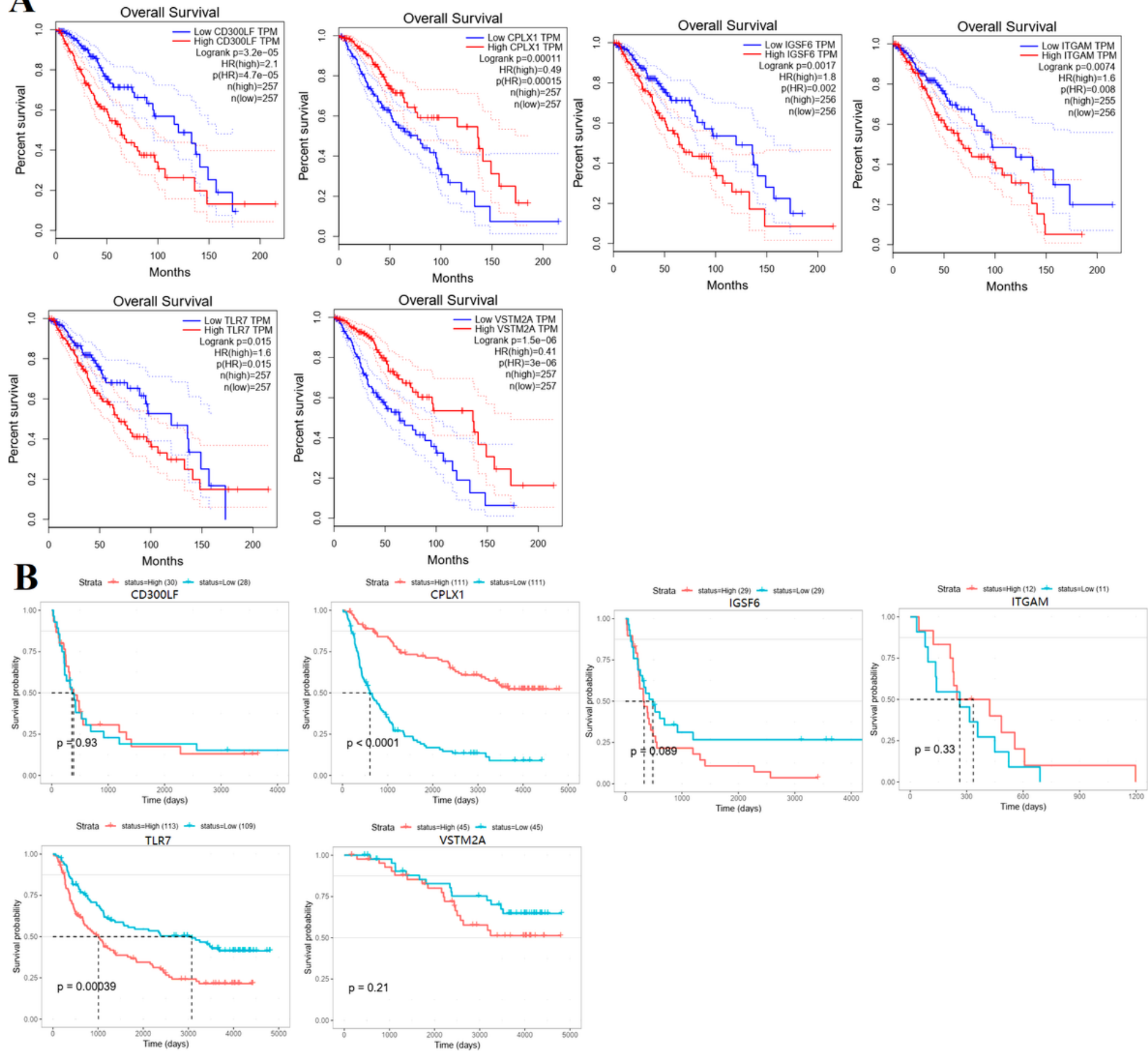

Figure 4

The survival analysis of the six hub genes based on TCGA and CGGA datebase. Kaplan-Meier plots overall survival of the six hub genes from TCGA datebase (A). The overall survival of the six hub genes were performed from CGGA datebase (B). 
A
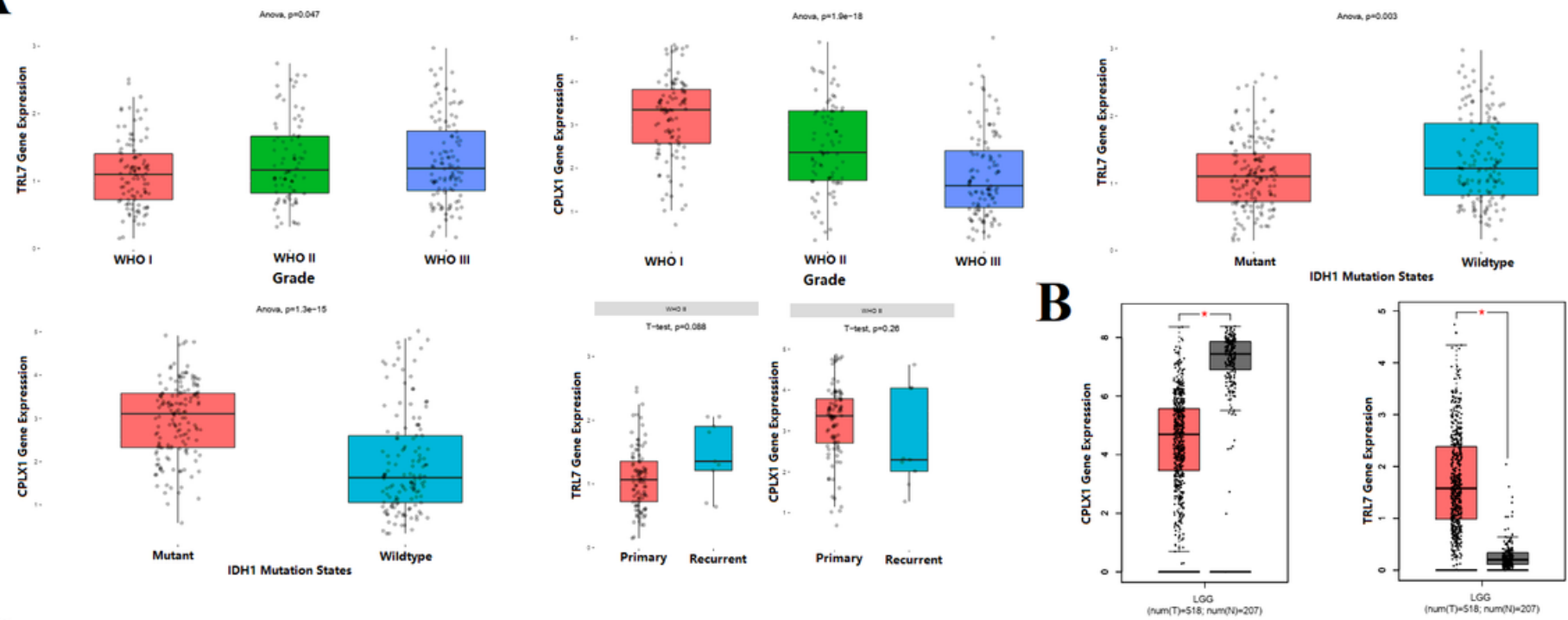

C
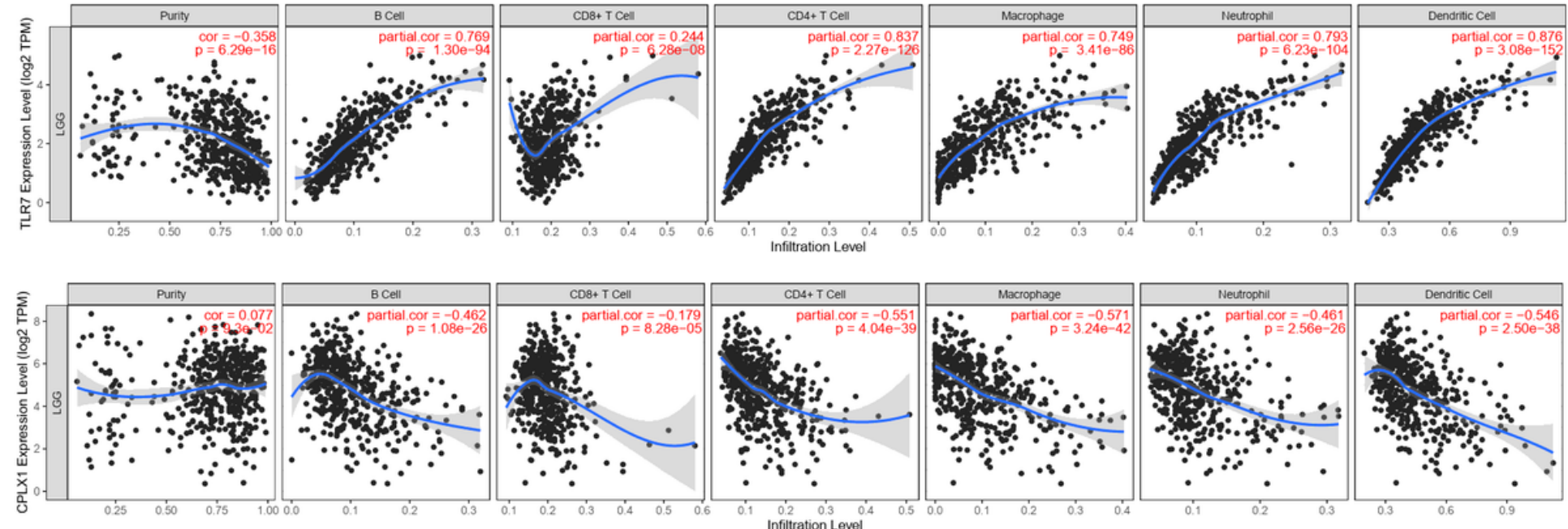

Figure 5

The relationship between hub genes and the clinicopathological characteristics of LGG patients(A). The different expression levels of hub genes expression between normal and turmor from TCGA(B). The correlation between hub genes expression and immune infiltration level in LGG via TIMER(C). 
A
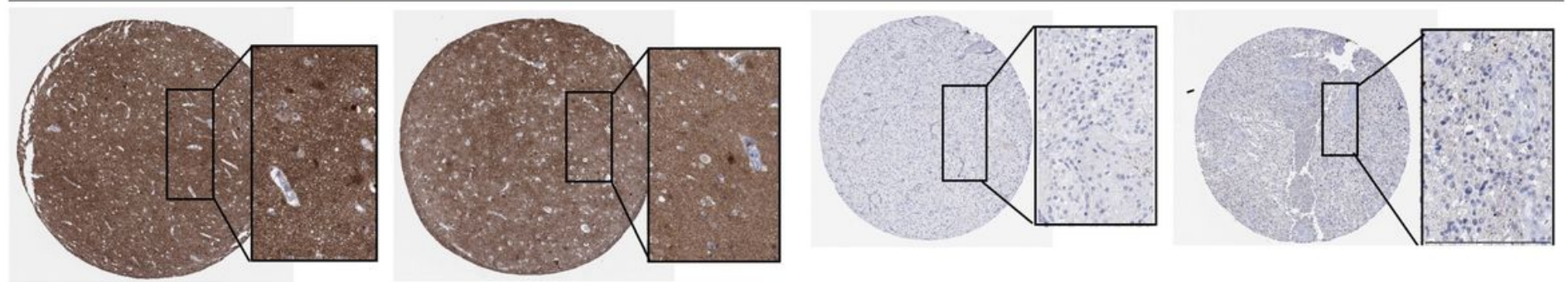

B

TRL7(N)

TRL7(T)
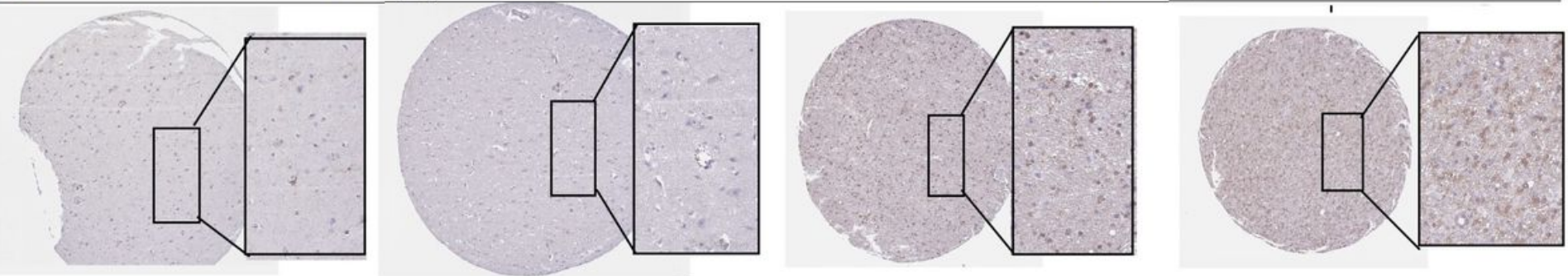

Figure 6

The protein expression and localization of CPLX1 and TLR7 in LGG by the Human Protein Expression Map Database.

\section{Supplementary Files}

This is a list of supplementary files associated with this preprint. Click to download.

- Figure3S1.tif

- Figure3S2.tif 